\title{
BUCKLING LOADS AND EIGENFREQUENCIES OF A BRACED BEAM RESTING ON AN ELASTIC FOUNDATION ${ }^{\star \star}$
}

\author{
Yin Zhang ${ }^{1 \star} \quad$ Yun Liu $^{2} \quad$ Peng Chen $^{3} \quad$ Kevin D Murphy ${ }^{4}$ \\ $\left({ }^{1}\right.$ State Key Laboratory of Nonlinear Mechanics (LNM), Institute of Mechanics, Chinese Academy of \\ Sciences, Beijing 100190, China) \\ $\left({ }^{2}\right.$ Faculty of Information and Automation, Kunming University of Science and Technology, Kunming \\ 650051, China) \\ ( ${ }^{3}$ Department of Civil Engineerning, Guangdong Technical College of Water Resources and Electric \\ Engineering, Guangzhou 510635, China) \\ ( ${ }^{4}$ Department of Mechanical Engineering, University of Connecticut, Storrs, CT 06269, USA)
}

Received 10 March 2011, revision received 21 June 2011

\begin{abstract}
The eigenvalue problems of the buckling loads and natural frequencies of a braced beam on an elastic foundation are investigated. The exact solutions for the eigenvalues are presented. The eigenvalues vary with the different parameters and are especially sensitive to the brace location. As the beam of a continuous system has infinite eigenvalues and these eigenvalues are influenced differently by a brace, the eigenvalues show rich variation patterns. Because these eigenvalues physically correspond to the structure buckling loads and natural frequencies, the study on the eigenvalues variation patterns can offer a design guidance of using a lateral brace of translation spring to strengthen the structure.
\end{abstract}

KEY WORDS beam, elastic foundation, buckling, eigenfrequency, curve crossing, curve veering

\section{INTRODUCTION}

In composite structures, the axial stress of a bending layer results in a stress in the direction normal to the interface due to the curvature effect, which is described by the famous Laplace-Young formula $^{[1]}$. In modeling aspect, the elastic foundation model is explicitly or implicitly introduced to describe the transverse interactions between layers ${ }^{[1-8]}$. The elastic foundation model simplifies the transverse interactions by assuming that the stress (normal to interface) at a point is proportional to its corresponding displacement. Otherwise, a rather complex formulation is needed ${ }^{[9]}$. With this transverse interaction, or say, the elastic foundation, the composite such as film/substrate structure can buckle with a large wave number ${ }^{[1-6]}$, which is also variably called wrinkling, undulation, convolution and ripple. Recently the large wave number wrinkling of thin film/substrate composite structures has been utilized to manufacture high-performance flexural electronics ${ }^{[10]}$ and to measure the thickness and Young's modulus of ultra-thin (nanometer scale) films ${ }^{[11]}$. On the other hand, as an important element in structural design, a lateral brace of translational spring with its variations of stiffness and

\footnotetext{
* Corresponding author. E-mail: zhangyin@lnm.imech.ac.cn

* Project supported by the National Natural Science Foundation of China(NSFC, Grant Nos. 10721202 and 11023001) and by Chinese Academy of Sciences(Grant No. KJX2-EW-L03).
} 
location has significant impact on the buckling loads, buckling shapes ${ }^{[12,13]}$ and eigenfrequencies ${ }^{[14,15]}$ of the structure. During the investigation of the buckling of cylindrical shells ${ }^{[16-18]}$, it was found that the characteristics of this type of structure can be well simulated by a column supported laterally by a spring.

Mathematically speaking, finding the buckling loads and eigenfrequencies of a system is an eigenvalue problem. The dependence of the eigenvalues upon the system parameters is often of interest in structural dynamics and design, which is frequently illustrated by a family of loci ${ }^{[19,20]}$. When two loci approach each other, they often cross (curve crossing) or abruptly diverge (curve veering) ${ }^{[20]}$. As for a beam on an elastic foundation with no brace, because the system buckles with the lowest buckling load, the curve crossings of the buckling loads physically correspond to the transition/change of buckling shapes $^{[21]}$. The curving veering phenomenon of eigenvalues exists in a variety of structural mechanics, such as vibration of beams ${ }^{[14,15,19]}$, rotors ${ }^{[22]}$, plates ${ }^{[23,24]}$ and shells ${ }^{[25]}$; buckling of columns ${ }^{[26]}$ and plates $^{[27]}$. However, in the early 1970 s, whether the occurrence of eigenvalue loci veering is a true physical phenomenon or a subtle manifestation due to mathematical approximation/discretization was intensively argued by Leissa ${ }^{[23]}$. Perkins and Mote ${ }^{[20]}$ showed that the veering indeed occurs in the exact solution for a rotating, guided, circular string; and at the same time, they also pointed out that some of the discretizing methods may destroy or create self-adjointness, which can affect the veering/crossing of the approximation solutions. The curve veering of eigenvalues in dynamics is often associated with the mode localization ${ }^{[19]}$, or say, the confinement of vibration energy ${ }^{[28]}$, which physically means that one portion of structure will experience much larger deflection (stress). Both the eigenvalue loci veering and mode localization are catastrophic because small changes of the system parameters cause large variations of eigenvalues and mode shapes, respectively ${ }^{[19]}$. Furthermore, when the two buckling loads come closer to each other an instability in the postbuckling region called mode-jumping, which is also catastrophic, will occur with less axial compression because of smaller difference between two buckling loads ${ }^{[29]}$.

The eigenvalues of a braced beam on an elastic foundation show rich variation patterns. Here the eigenvalues are solved exactly without any discretization process. The eigenvalues are shown to be sensitive to some system parameters. Besides the curve crossings, the eigenvalue loci are also found to veer away from each other as the brace moves from one end to the other. However, we also show that the curve crossing or curve veering of the eigenvalue loci can be avoided with small variation of parameters. Some of the eigenvalue loci variation patterns and trends are observed and summarized, which may serve as a helpful guidance to the structural design.

\section{EQUATIONS OF EQUILIBRIUM AND BUCKLING LOADS}

Figure 1 is a schematic diagram of a hinged-hinged beam resting on an elastic foundation; the beam is braced laterally with a translational spring with the stiffness of $k_{s}$. The brace locates at $x=a$ and $k$ is the elastic foundation modulus. $E$ and $I$ are the Young's modulus and area moment of inertia of the beam, respectively. $L$ is the beam length. $p$ is the axial load; here positive $p$ indicates compression and negative $p$ is tension. $w$ is the beam deflection, which is divided into two parts as follows:

$$
w= \begin{cases}w_{1} & (0 \leq x<a) \\ w_{2} & (a \leq x \leq L)\end{cases}
$$

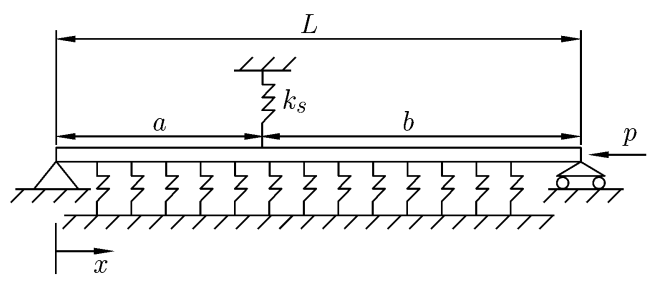

Fig. 1. Schematic diagram of a braced beam resting on an elastic foundation. 
The governing equations for these two parts are

$$
\begin{array}{ll}
E I \frac{\mathrm{d}^{4} w_{1}}{\mathrm{~d} x^{4}}+p \frac{\mathrm{d}^{2} w_{1}}{\mathrm{~d} x^{2}}+k w_{1}=0 & (0 \leq x<a) \\
E I \frac{\mathrm{d}^{4} w_{2}}{\mathrm{~d} x^{4}}+p \frac{\mathrm{d}^{2} w_{2}}{\mathrm{~d} x^{2}}+k w_{2}=0 & (a \leq x \leq L)
\end{array}
$$

The boundary conditions are the following:

$$
w_{1}(0)=0, \quad \frac{\mathrm{d}^{2} w_{1}}{\mathrm{~d} x^{2}}(0)=0, \quad w_{2}(L)=0, \quad \frac{\mathrm{d}^{2} w_{2}}{\mathrm{~d} x^{2}}(L)=0
$$

The matching/transition conditions at $x=a$ are as follows ${ }^{[14]}$ :

$$
w_{1}(a)=w_{2}(a), \quad \frac{\mathrm{d} w_{1}}{\mathrm{~d} x}(a)=\frac{\mathrm{d} w_{2}}{\mathrm{~d} x}(a), \quad \frac{\mathrm{d}^{2} w_{1}}{\mathrm{~d} x^{2}}(a)=\frac{\mathrm{d}^{2} w_{2}}{\mathrm{~d} x^{2}}(a), \quad \frac{\mathrm{d}^{3} w_{1}}{\mathrm{~d} x^{3}}(a)=\frac{\mathrm{d}^{3} w_{2}}{\mathrm{~d} x^{3}}(a)+\frac{k_{s}}{E I} w_{1}(a)
$$

The effect of the bracing spring is now incorporated in the above matching conditions. An alternative is to include the bracing spring into the governing equation as follows:

$$
E I \frac{\mathrm{d}^{4} w}{\mathrm{~d} x^{4}}+p \frac{\mathrm{d}^{2} w}{\mathrm{~d} x^{2}}+k w+k_{s} w \delta(x-a)=0
$$

$\delta$ here is the Dirac delta function. It is extremely difficult if not impossible to give an analytical solution to Eq.(5). However, by breaking $w$ into two parts as done in Eq.(2), the solutions are readily given as follows:

$$
w_{i}(x)=A_{i} \cos \left(\gamma_{1} x\right)+B_{i} \sin \left(\gamma_{1} x\right)+C_{i} \cos \left(\gamma_{2} x\right)+D_{i} \sin \left(\gamma_{2} x\right) \quad(i=1,2)
$$

$A_{i}, B_{i}, C_{i}$ and $D_{i}$ are the constants to be determined. $\gamma_{1}$ and $\gamma_{2}$ are defined as

$$
\gamma_{1}=\sqrt{\alpha-\sqrt{\alpha^{2}-\beta^{2}}}, \quad \gamma_{2}=\sqrt{\alpha+\sqrt{\alpha^{2}-\beta^{2}}} \quad\left(\alpha=\frac{p}{2 E I}, \quad \beta=\sqrt{\frac{k}{E I}}\right)
$$

Now there are eight unknowns $A_{i}, B_{i}, C_{i}, D_{i}(i=1,2)$. Equations (3) and (4) give eight boundary and transition conditions in total. To have nontrivial solutions for Eq.(6), the determinant of the $8 \times 8$ matrix resulting from Eqs.(3) and (4) needs to be zero. The eigenvalues of buckling loads are thus solved by setting the determinant to be zero. Here we need to point out that the solution of Eq.(6) assumes $\alpha^{2}-\beta^{2}>0$. If $\alpha^{2}-\beta^{2} \leq 0$, the solution form will be very different from Eq.(6). However, if $\alpha^{2}-\beta^{2} \leq 0$ is assumed, there are no solutions for buckling loads in the real domain.

\section{EQUATIONS OF MOTION AND EIGENFREQUENCIES}

The equation of motion which does not include damping effect is given as follows:

$$
\begin{array}{cl}
M \ddot{w}_{1}+E I \frac{\mathrm{d}^{4} w_{1}}{\mathrm{~d} x^{4}}+p \frac{\mathrm{d}^{2} w_{1}}{\mathrm{~d} x^{2}}+k w_{1}=0 & (0 \leq x<a) \\
M \ddot{w}_{2}+E I \frac{\mathrm{d}^{4} w_{2}}{\mathrm{~d} x^{4}}+p \frac{\mathrm{d}^{2} w_{2}}{\mathrm{~d} x^{2}}+k w_{2}=0 & (a \leq x \leq L)
\end{array}
$$

Here $M$ is the beam mass per unit length; $\ddot{(})=\mathrm{d}^{2} / \mathrm{d} t^{2}(t$ is time). Unlike $p$ in Eq. $(5)$ which is the buckling load to be determined from the eigenvalue problem, in Eq.(8) $p$ is a given axial load. Assuming $w_{i}(x, t)=\phi(t) v_{i}(x)(i=1,2)$ and by separation of variables, we have the following governing equation:

$$
\begin{array}{ll}
E I \frac{\mathrm{d}^{4} v_{1}}{\mathrm{~d} x^{4}}+p \frac{\mathrm{d}^{2} v_{1}}{\mathrm{~d} x^{2}}+\left(k-M \Omega^{2}\right) v_{1}=0 & (0 \leq x<a) \\
E I \frac{\mathrm{d}^{4} v_{2}}{\mathrm{~d} x^{4}}+p \frac{\mathrm{d}^{2} v_{2}}{\mathrm{~d} x^{2}}+\left(k-M \Omega^{2}\right) v_{2}=0 & (a \leq x \leq L)
\end{array}
$$

Here $\Omega^{2}=-\ddot{\phi}(t) / \phi(t)$ and $\Omega$ is the eigenfrequency to be determined. Again, we encounter the problem of determining the solution form: $k-M \Omega^{2}<0, k-M \Omega^{2}=0$ and $k-M \Omega^{2}>0$ will give three different solution forms for Eq.(9). $k-M \Omega^{2}<0$, i.e., $\Omega>\sqrt{k / M}$ is the cut-on frequency ${ }^{[30,31]} ; \Omega=\sqrt{k / M}$ 
is the cut-off frequency ${ }^{[30]} ; \Omega<\sqrt{k / M}$ is the case of the frequency below the cut-off frequency, which is also referred to as the divergence instability ${ }^{[14]}$. Reference [31] presents a proof that only the cuton frequency can exist for a finite length beam resting on an elastic foundation. Because the cut-on frequency is the only frequency for the eigenfrequency for the system, the solution forms of Eq.(9) are given as follows:

$$
v_{i}(x)=A_{i} \cosh \left(\lambda_{1} x\right)+B_{i} \sinh \left(\lambda_{1} x\right)+C_{i} \cos \left(\lambda_{2} x\right)+D_{i} \sin \left(\lambda_{2} x\right) \quad(i=1,2)
$$

Here $A_{i}, B_{i}, C_{i}$ and $D_{i}(i=1,2)$ are the eight constants to be determined. $\lambda_{1}$ and $\lambda_{2}$ are defined as

$$
\lambda_{1}=\sqrt{\alpha-\sqrt{\alpha^{2}-\beta^{* 2}}}, \quad \lambda_{2}=\sqrt{\alpha+\sqrt{\alpha^{2}-\beta^{* 2}}} \quad\left(\alpha=\frac{p}{2 E I}, \quad \beta^{*}=\sqrt{\frac{M \Omega^{2}-k}{E I}}\right)
$$

Once again, the eight boundary and transition conditions given by Eqs.(3) and (4) form an eigenvalue problem for $\Omega$. The eigenvalue problems of the buckling and eigenfrequencies are different due to the different solution forms of Eqs.(6) and (10).

\section{RESULTS AND DISCUSSIONS}

Before examining the effect of the bracing spring, some of the buckling characteristics of the beam on an elastic foundation without a brace need to be outlined. The buckling loads of a hinged-hinged beam on an elastic foundation with no brace is given by Timoshenko and Gere ${ }^{[21]}$ as follows:

$$
p_{m}=\frac{\pi^{2} E I}{L^{2}}\left(m^{2}+\frac{k L^{4}}{m^{2} \pi^{2} E I}\right)
$$

Here $m$ is an integer. $p_{m}$ is the buckling load of the $m$ th buckling shape of $\sin (m \pi x / L)$ for a hingedhinged beam on an elastic foundation ${ }^{[21]}$. The plot of beam length $L$ versus $p_{m}$ is shown in Fig.2; here the beam bending stiffness $E I$ and elastic foundation modulus $k$ are both set as $1^{[21]}$. As shown in Fig.2, the curves of $p_{1}$ and $p_{2}$ intersect at $L=4.45$. After the intersection, the lowest buckling load changes from $p_{1}$ to $p_{2}$; the buckling shape also changes correspondingly from $\sin (\pi x / L)$ to $\sin (2 \pi x / L)$. At $L=7.7$, the lowest buckling load then changes from $p_{2}$ to $p_{3}$. At $L=10.86$, the lowest buckling load changes from $p_{3}$ to $p_{4}$. At $L=14.04$, the lowest buckling load changes from $p_{4}$ to $p_{5}$ and so on. Those transition/intersection points are all marked with triangles in Fig.2. When the lowest buckling load curve changes, the buckling shape also shifts correspondingly as well as the wave number. However, there is no coupling between the buckling shapes of $\sin (m \pi x / L)$ during the transitions of the lowest

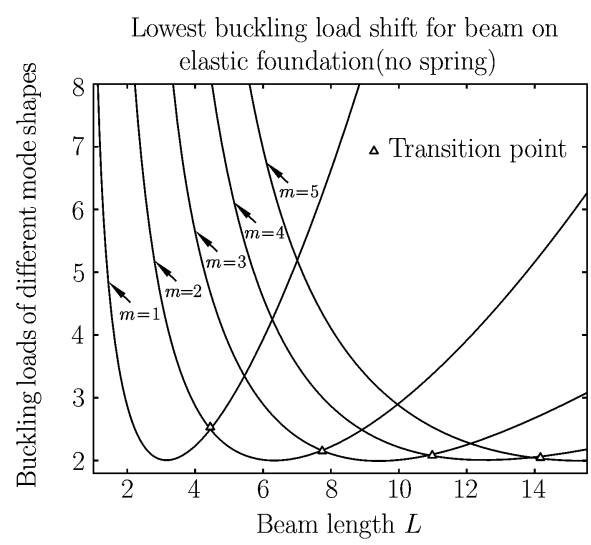

Fig. 2. Buckling loads associated with different buckling shapes of $\sin (m \pi x / L)$ for an unbraced beam on an elastic foundation. The transition points are marked with triangles.

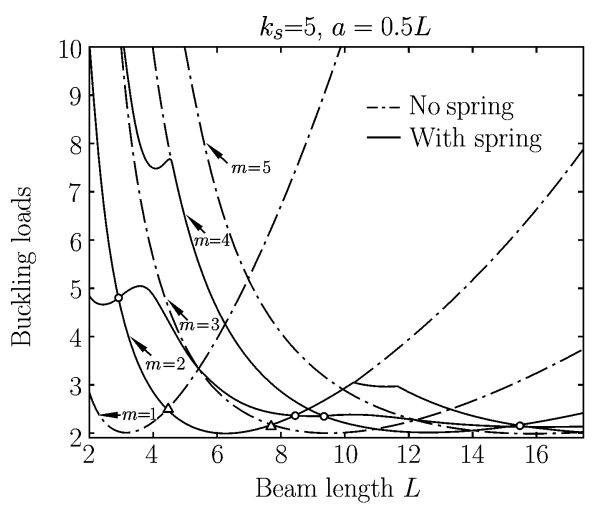

Fig. 3. The three lowest buckling loads of the braced beam compared with those of the unbraced one as a function of the beam length $L$. The brace is placed at the beam center of $a=0.5 L$ and its spring stiffness is $k_{s}=5$. The transition points of the braced beam are marked with circles and two transition points of the unbraced beam are marked with triangles. 
buckling load curves. Although the beam buckles with a fixed wave number $m \pi$ at different $L$ (except those transition points), those beams with the buckling loads close to the transition points will have greater danger of experiencing the mode-jumping instability in the post-buckling region ${ }^{[29]}$. When the axial load reaches a critical value in the post-buckling region, the beam can no longer keep its previous buckling shape and the beam has a sudden change of its deflection shape and wave number ${ }^{[29]}$, which can cause significant damage to the structure. So for this kind of structure, the structure buckling loads should be designed to be away from those transition points.

Figure 3 shows the case that a bracing spring is located at the beam center of $a=0.5 L$, which is also the node of $\sin (2 \pi x / L)$ (i.e., $\sin (2 \pi a / L)=\sin (\pi)=0$ ). The bracing spring stiffness is $k_{s}=5$. Three lowest buckling loads are computed and compared with those of the unbraced ones. Clearly from Fig.3, the lowest buckling load increases significantly for short beams. For example, at $L=2$, the lowest buckling load of the braced beam is 4.87 compared with 2.873 of the unbraced beam. Also for the braced beam, the transition occurs at a smaller length of $L=2.94$ (the transition points for the braced beam are marked with circles) compared with $L=4.45$ of the unbraced beam (marked with a triangle). After the intersection at $L=2.94$, the lowest buckling load of the braced beam overlaps with the $p_{2}$ curve of the unbraced beam. It is worth noticing that between the two transition points of $L=4.45$ and $L=7.7$ for the unbraced beam (marked with triangles), the lowest buckling loads of the braced and unbraced beams are (almost) the same. With the increase of beam length, the difference between the lowest buckling loads of the braced and unbraced beams becomes smaller, which physically means that the bracing spring has little impact on the lowest system buckling load for a very long beam. Unlike the unbraced beam of no coupling between the buckling shapes of $\sin (m \pi x / L)$, the buckling shape of the braced beam is a coupled one. The buckling shape at $L=2, k_{s}=5$ and $p=4.87$ is shown in Fig.4. In Fig.4, the buckling shape is asymmetric. This asymmetry can be thought of as the result from the coupling of $\sin (m \pi x / L) \cdot \sin (m \pi x / L)$ is the mode shape of the unbraced beam, which is symmetric when $m$ is odd and antisymmetric when $m$ is even. The prensence of the brace in essence couples these symmetric and antisymmetric mode shapes and leads to an asymmetric one. Figure 5 shows the case that a brace is also located at $a=0.5 L$ but with a much larger stiffness of $k_{s}=50$. With this large bracing spring stiffness, starting from $L=2$, the lowest buckling load of the braced beam overlaps the $p_{2}$ curve of the unbraced beam. Therefore, for small $L$ (e.g. $L<4$ ), the lowest buckling load is significantly increased. Again, the same scenario as that in Fig. 3 occurs: in the range of $4.45 \leq L \leq 7.7$, the lowest buckling loads of the braced and unbraced beams are the same. It is noticed that the curve crossing is postponed until $L=8.932$ (marked with a circle) and more interestingly, the three lowest buckling loads of the braced beam intersect (almost) exactly at the same one point; their next curve crossing occurs at $L=15.475$. Once again, we observe that there is no significant difference between the lowest buckling of the braced beam and that of the unbraced one when the beam length is large (e.g. $L>15)$.

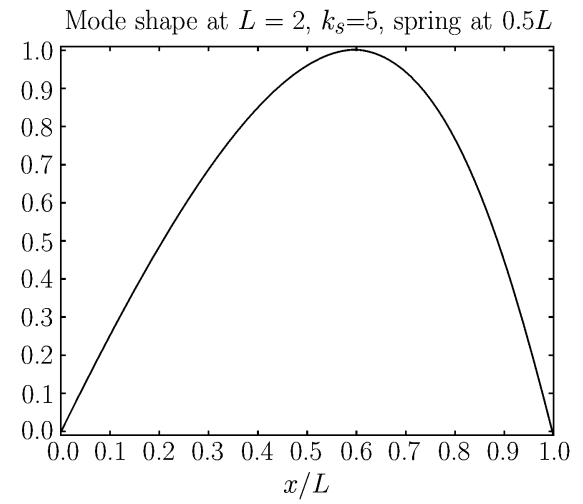

Fig. 4. The buckling shape of the braced beam with $k_{s}=5, a=0.5 L$ and $L=2$.

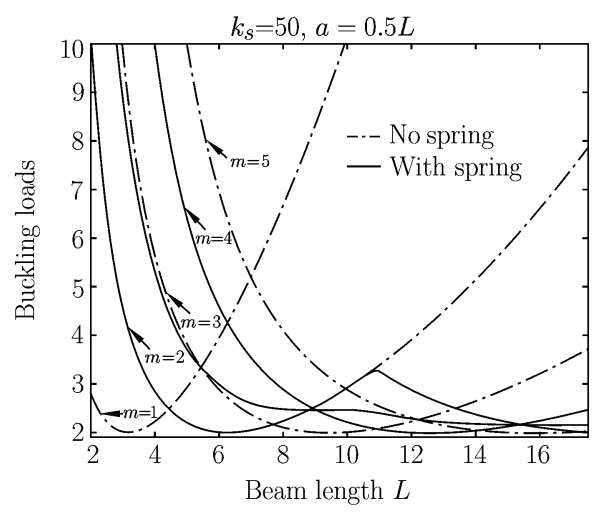

Fig. 5. The three lowest buckling loads of the braced beam compared with those of the unbraced one as a function of the beam length $L$. The brace is placed at the beam center and its spring stiffness is $k_{s}=50$. 


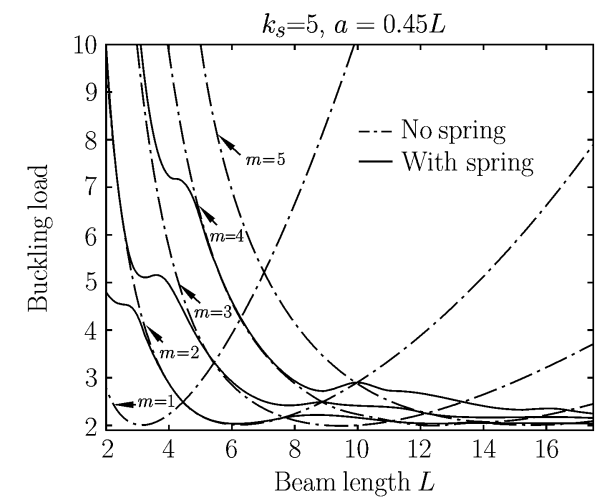

Fig. 6. The three lowest buckling loads of the braced beam compared with those of the unbraced one as a function of the beam length $L$. The brace is placed at $a=0.45 L$ and its spring stiffness is $k_{s}=5$.

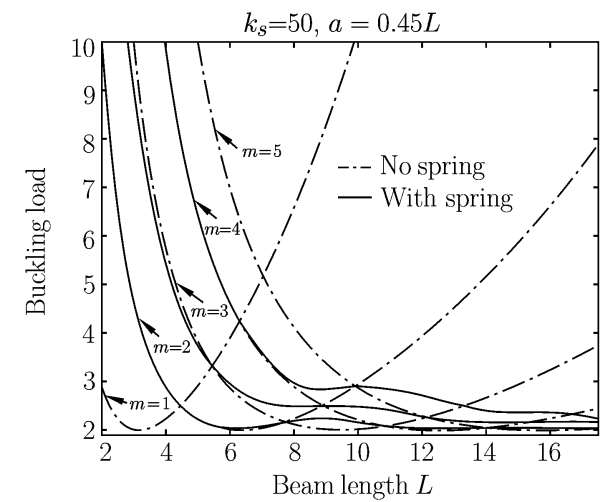

Fig. 7. The three lowest buckling loads of the braced beam compared with those of the unbraced one as a function of the beam length $L$. The brace is placed at $a=0.45 L$ and its spring stiffness is $k_{s}=50$.

Figures 6 and 7 show the cases when the brace shifts a little from the center to $a=0.45 L . k_{s}$ is still taken as 5 and 50. Compared with the cases of the brace located at the center shown in Figs. 3 and 4, some similar trends are also observed in Figs.5 and 6: (1) the brace improves the lowest buckling load of the short beam; (2) some portions of the lowest buckling loads of the braced and unbraced beams overlap; (3) the impact of the brace on the lowest buckling load reduces with the increase of the beam length. The biggest difference between Figs. 6 and 7 (cases of brace off the center) and Figs. 3 and 5 (cases of brace at the center) is that the three lowest buckling loads in Figs.6 and 7 are well separated from each other and no curve crossing occurs. This well separated buckling loads will be helpful to delay or even eliminate a post-buckling instability called mode jumping which is directly associated with the 'closeness' of two neighboring buckling loads ${ }^{[29]}$. If the brace is further shifted to $a=L / 3$ and $k_{s}$ is kept as $k_{s}=50$, the three lowest buckling loads are shown in Fig.8. The beam buckles at the mixed modes shape until $L=6.75$ and again, the lowest buckling load of short beam increases significantly compared with that of the unbraced one. From $L=6.75$ to $L=11.58$, the lowest buckling load with the braced beam overlaps the $p_{3}$ curve (with the buckling shape of $\sin (3 \pi x / L)$ ) of the unbraced one. And from $L=11.58$ the lowest buckling load of the braced case separates from $p_{3}$ and becomes a mixed mode buckling again. At $L=16.4$, the lowest buckling load of the braced case merges with the $p_{5}$ curves. From that point, the lowest buckling of braced case converges to that of no brace case.

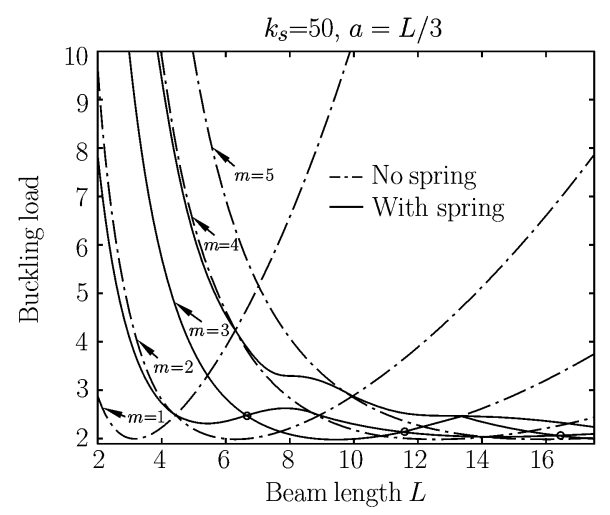

Fig. 8. The three lowest buckling loads of the braced beam compared with those of the unbraced one as a function of the beam length $L$. The brace is placed at $a=L / 3$ and its spring stiffness is $k_{s}=50$.

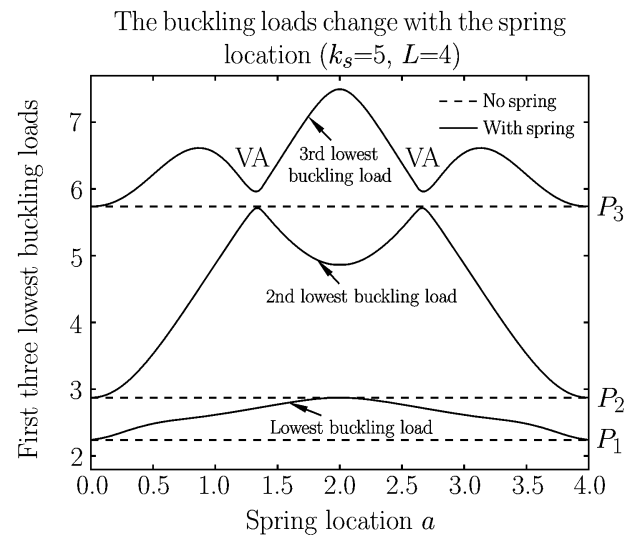

Fig. 9. The three lowest buckling loads of the braced beam with $L=4$ and $k_{s}=5$ as a function of the brace location $a$. The buckling loads of the unbraced beam are plotted as dashed straight lines. VA stands for veering away. 


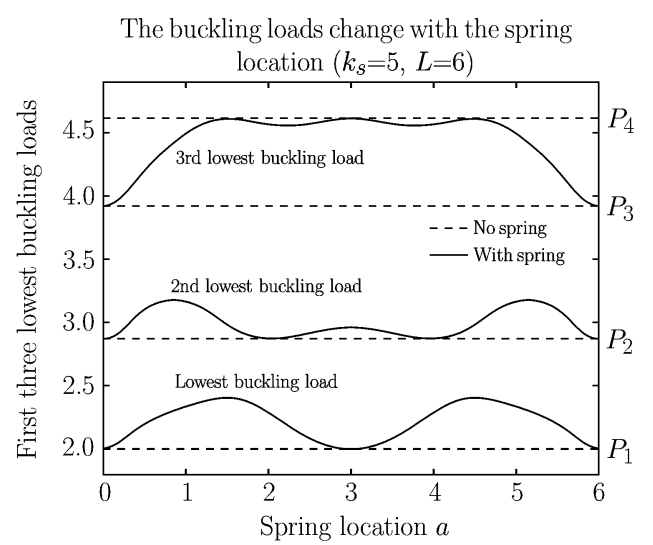

Fig. 10. The three lowest buckling loads of the braced beam with $L=6$ and $k_{s}=5$ as a function of the brace location $a$. The four lowest buckling loads of the unbraced beam are plotted as dashed straight lines.

Now let us look from another different angle at the effect of the brace on the beam structure buckling loads, which may show us some other trends/rules. In Fig.9, the beam length is fixed as $L=4$, the brace spring stiffness is $k_{s}=5$ and the brace moves from one end to the the other. Figure 9 shows that the three lowest buckling loads of the braced structure change as the brace moves from $a=0$ to $a=L$. For comparison, the three lowest buckling loads of the unbraced beam are also plotted together as dashed straight lines, which are calculated from Eq.(12) as $p_{1}=2.238, p_{2}=2.872$ and $p_{3}=5.7318$ $\left(p_{1}<p_{2}<p_{3}\right)$. In Fig.9, it is noticed that when the brace is at the center, the lowest buckling equals the $p_{2}$ value. This is also reflected in Fig. 3, where at $L=4$ the lowest buckling load of the braced beam overlaps the $p_{2}$ curve. When the brace is located at the center, the first and the third lowest buckling loads reach their maximum; the second lowest buckling load reaches its minimum. The reason is that $x=L / 2$ is the peak of both $\sin (\pi x / L)$ and $\sin (3 \pi x / L)$, which can contributes most to improve their corresponding buckling load; however, $x=L / 2$ is the node of $\sin (2 \pi x / L)$, which correspondingly does the least help to improve its buckling load. This impact of the brace location reminds us the very similar effect of the location of quantum dots on the local bending of a thin film ${ }^{[8]}$. It is also noticed that in Fig.9 at $a=L / 3$ and $a=2 L / 3$, the second and third lowest buckling loads approach and diverge abruptly, which is the 'veer away (VA)' case of eigenvalues ${ }^{[20]}$. Now we change the beam length as $L=6$ and $k_{s}$ is still fixed as $k_{s}=5$ to see how the three lowest buckling loads of the braced behave. Again, the four lowest buckling loads of the unbraced beam calculated from Eq.(12) are plotted for comparison reason: $p_{2}=2.0, p_{3}=2.87, p_{1}=3.92$ and $p_{4}=4.61$. Now the sequence is $p_{2}<p_{3}<p_{1}<p_{4}$ in Fig.10. Unlike those in Fig.9, the three lowest buckling loads of the braced beam in Fig.10 are now well separated. When the brace is at the center, the lowest buckling equals the $p_{2}$ value of 2.0 , which is also reflected in Fig. 3 where the lowest buckling load curve of the braced beam overlaps the $p_{2}$ curve at $L=6$. The reason for the first lowest buckling in Fig.10 to reach its minimum at the center is that $x=L / 2$ is the node of $\sin (2 \pi x / L)$ and the brace does not do any help to improve the buckling load. This, again, verifies the fact that the brace at some point does not contribute anything to increase the structure lowest buckling load.

Figures 11 and 12 examine the impact of the brace on the structure eigenfrequencies. In both figures the beam length $L$ is set as 2 ; the axial load $p$ is set as 1 (much smaller than the buckling load); the brace stiffness $k_{s}$ varies from $k_{s}=0$ to $k_{s}=50$. In Fig.11, the brace is located at $0.5 L, 0.475 L$ and $0.45 \mathrm{~L}$, respectively. Clearly, for the eigenfrequencies of the second mode, there are no or much less changes than those of first and third modes. Because $x=0.5 \mathrm{~L}$ is the node of the second mode shape of $\sin (2 \pi x / L)$, the brace stiffening effect at this exact location is zero. In Fig.12, the brace is located at $L / 3,0.33 L$ and $0.3 L$. Clearly it is shown that there are no or much less eigenfrequency changes for the third mode shape of $\sin (3 \pi x / L)$ than those of first and second mode shapes. The reason, again, is the same: $x=L / 3$ is the node of $\sin (3 \pi x / L)$. 


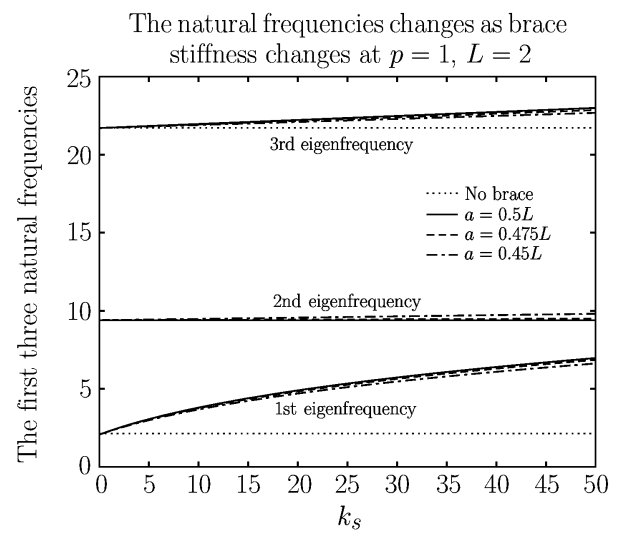

Fig. 11. The first three natural frequencies of the braced beam with $a=0.5 L, 0.475 L$ and $0.45 L$ as a function the brace stiffness $k_{s}$.

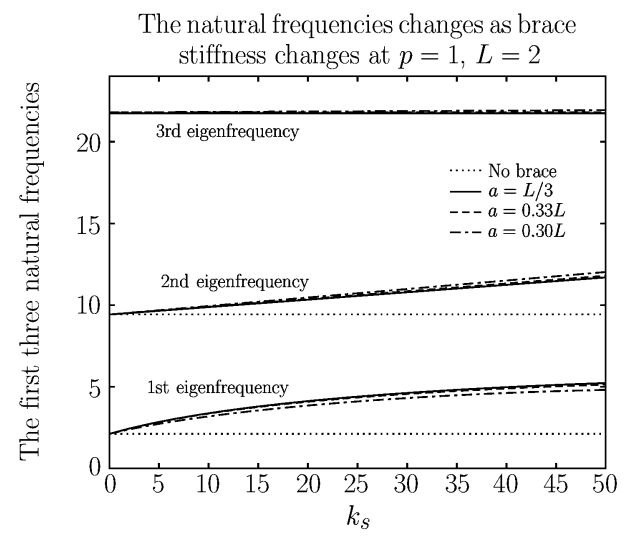

Fig. 12. The first three natural frequencies of the braced beam with $a=L / 3,0.33 L$ and $0.3 L$ as a function the brace stiffness $k_{s}$.

\section{CONCLUSION}

The eigenvalues (buckling loads and eigenfrequencies) of the braced beam on an elastic foundation are solved exactly with the variations of different system parameters. The eigenvalues are sensitive to the spring stiffness and location of the brace. The brace location plays a more important role, which changes not only the eigenvalue magnitude but also its variation pattern (curve crossing and veering). For a hinged-hinged braced beam, when the brace locates around/at the nodes of $\sin (m \pi x / L$ ) (which is the buckling shape or the mode shape of an unbraced beam), the brace has little or no contribution to the system eigenvalues; when the brace locates around/at the peaks/valleys of $\sin (m \pi x / L)$, the brace contributes most to increase the system eigenvalues. With the increase of the beam length, the influence of the brace is reduced and the lowest buckling load of the braced beam converges to that of the unbraced beam.

\section{References}

[1] Suo,Z., Wrinkling of the oxide scale on an aluminum-containing alloy at high temperatures. Journal of the Mechanics and Physics of Solids, 1995, 43: 829-846.

[2] Sridhar,N., Srolovitz,D.J. and Suo,Z., Kinetics of buckling of a compressed film on a viscous substrate. Applied Physics Letters, 2001, 78: 2482-2484.

[3] Huang,R. and Suo,Z., Instability of a compressed elastic film on a viscous layer. International Journal of Solids and Structures, 2002, 39: 1791-1802.

[4] Huang,R. and Suo,Z., Wrinkling of a compressed elastic film on a viscous layer. Journal of Appllied Physics, 2002, 91: 1135-1142.

[5] Bowden,N., Brittain,S., Evans,A.G., Hutchinson,J.W. and Whitesides,G.M., Spontaneous formation of ordered structures in thin films of metals supported on an elastomeric polymer. Nature, 1998, 393: 146-149.

[6] Jiang,H., Sun,Y., Rogers,J.A. and Huang,Y., Post-buckling analysis for the precisely controlled buckling of thin film encapsulated by elastomeric substrates. International Journal of Solids and Structures, 2008, 45: 2014-2023.

[7] Zhang,Y. and Zhao,Y., Discussion on modeling shape memory alloy embedded in a composite laminate as axial force and elastic foundation. Materials and Design, 2007, 28: 1016-1020.

[8] Zhang,Y. and Liu,Y., Local bending of thin film on viscous layer. Acta Mechanica Solida Sinica, 2010, 23: 106-114.

[9] Zhang,Y. and Zhao,Y., A study of composite beam with shape memory alloy arbitrarily embedded under thermal and mechanical loadings. Materials and Design, 2007, 28: 1096-1115.

[10] Khang,D., Jiang,H., Huang,Y. and Rogers,J.A., A stretchable form of single crystal silicon for highperformance electronics on rubber substrates. Science, 2006, 311: 208-212.

[11] Huang,J., Juszkiewicz,M., de Jeu,W.H., Cerda,E., Emrick,T., Menon,N. and Russel,T., Capillary wrinkling of floating thin films. Science, 2007, 317: 650-653.

[12] Plaut,R.H. and Yang,J.G., Lateral bracing forces in columns with two unequal spans. Journal of Structural Engineering, 1992, 119: 2896-2912. 
[13] Plaut,R.H., Requirements for lateral bracing of columns with two spans. Journal of Structural Engineering, 1992, 119: 2913-2931.

[14] Plaut,R.H, Murphy,K.D. and Virgin,L.N., Curve and surface veering for a braced column. Journal of Sound and Vibration, 1995, 187: 879-885.

[15] Chen,P.T. and Ginsberg,J.H., On the relationship between veering of eigenvalue loci and parameter sensitivity of eigenfunctions. Journal of Vibration and Acoustics, 1992, 114: 141-148.

[16] von Kármán,T., Dunn,L.G. and Tsien,H., The influence of curvature on the buckling characteristics of structures. Journal of the Aeronautical Sciences, 1940, 7: 276-289.

[17] Tsien,H., A theory of the buckling of thin shells. Journal of the Aeronautical Sciences, 1942, 9: 373-384.

[18] Hutchinson,J.W. and Budiansky,B., Dynamic buckling estimates. AIAA Journal, 1966, 4: 525-530.

[19] Pierre,C., Mode localization and eigenvalue loci veering phenomena in disordered structures. Journal of Sound and Vibration, 1988, 126: 485-502.

[20] Perkins,N.C. and Mote,C.D., Comments on curve veering in eigenvalue problems. Journal of Sound and Vibration, 1986, 106: 451-463.

[21] Timoshenko,S. and Gere,J.M., Theory of Elastic Stability. McGraw-Hill Book Company, Inc., 1981.

[22] Jei,Y.G. and Lee,C.W., Does curve veering occur in the eigenvalue problem of rotors? Journal of Vibration and Acoustics, 1992, 114: 32-36.

[23] Leissa,A.W., On a curve veering aberration. Zeitschrift für Angewandte Mathematik und Physik(ZAMP), 1974, 25: 99-111.

[24] Kutter,J.R. and Sigillito,V.G., On curve veering. Journal of Sound and Vibration, 1981, 75: 585-588.

[25] Chen,P.T. and Ginsberg,J.H., Modal properties and eigenvalue veering phenomena in axisymmetric vibration of spheroidal shells. Journal of the Acoustic Society of America, 1992, 92: 1499-1508.

[26] Pierre,C. and Plaut,R.H., Curve veering and mode localization in a buckling problem. Zeitschrift für Angewandte Mathematik und Physik(ZAMP), 1989, 40: 758-761.

[27] Wang,J. and Mote,C.D., On the divergence buckling of a wide bandsaw plate by roll-tensioning. Journal of Sound and Vibration, 1986, 175: 661-675.

[28] Castanier,M.P. and Pierre,C., Predicting localization via Lyaponov exponent statistics. Journal of Sound and Vibration, 1997,203, 151-157.

[29] Zhang,Y. and Murphy,K.D., Secondary buckling and tertiary states of a beam on a non-linear elastic foundation. International Journal of Nonlinear Mechanics, 2005, 40: 795-805.

[30] Graff,K.F., Wave Motion in Elastic Solids, Ohio State University Press, 1975.

[31] Zhang,Y. and Murphy,K.D., Crack propagation in structures subjected to periodic excitation. Acta Mechanica Solida Sinica, 2007, 20: 230-240. 